\title{
Eficacia de los ácidos grasos hiperoxigenados en la prevención de las úlceras por presión
}

\section{Hyperoxygenated fatty acid effectiveness in the prevention of the pressure ulcers}

\author{
Alonso López Escribano \\ Francisco García Alcaraz \\ Patricia Jareño Collado \\ José García Aranos \\ Nieves García Piqueras
}

\author{
Enfermero, coordinador de equipos de Enfermería Gerencia Atención Primaria del Área \\ de Salud de Albacete (SESCAM). \\ Enfermero, profesor asociado Enfermería Comunitaria. EU de Enfermería Albacete. \\ Enfermero del centro de salud de Alcadozo y en la Unidad de Investigación, Docencia y \\ Formación de la Gerencia Atención Primaria del Área de Salud de Albacete (SESCAM). \\ Farmacéutica. \\ Enfermero, coordinador de equipos de Enfermería Gerencia Atención Primaria del Área \\ de Salud de Albacete (SESCAM). \\ Enfermera, directora de Enfermería Gerencia Atención Primaria del Área de Albacete \\ (SESCAM).
}

Institución: Gerencia Atención de Atención Primaria del Área de Salud de Albacete (SESCAM).

\author{
Correspondencia: \\ Alonso López Escribano \\ Sescam-Gerencia Atención Primaria de Albacete \\ C/ Marqués de Villores, 6-8 \\ 02001-Albacete \\ Tfno.: 675831107 / 638228055 \\ E-mail: alonsol@sescam.jccm.es
}

\section{RESUMEN}

Objetivos: Conocer la eficacia de los ácidos grasos hiperoxigenados en la prevención de las úlceras por presión. Determinar el nivel de evidencia del uso de los ácidos grasos hiperoxigenados en la prevención de las úlceras por presión. Material y método: Se realizó una búsqueda bibliográfica en Cochrane library, The Joanna Briggs Institute, Pubmedmedline y Cuiden, siendo solamente seleccionados ensayos clínicos. Para su evaluación se han seguido las recomendaciones del Critical Appraisal Skills Programme (CASPe) junto al sistema GRADE para la valoración de la calidad de la evidencia y la fuerza de las recomendaciones.

Resultados: En the Cochrane Library no aparece ningún documento, mientras que en The Joanna Briggs Institute aparece un bestpractice donde no se hace ninguna referencia a los AGHO. En el resto de bases encontramos 2 ensayos clínicos aleatorizados, que cumplían los criterios CASPe. En el estudio de Gallart, et al. sobre una muestra de 192 pacientes se observó una incidencia de UPP del 19\% en el grupo intervención frente al 35\% del grupo placebo $(\mathrm{p}<0,007)$, siendo la RAR de $16(3,9-28,1)$, la RRR de 45,7 $(11,8-66,6)$ y el NNT fue de 7 (4-26), con un intervalo de confianza del $95 \%$. Por su parte, Torra i Bou, et al., sobre una muestra de 331 pacientes, observaron una incidencia de UPP del $7,32 \%$ en el grupo intervención frente a $17,37 \%$ en el grupo placebo $(\mathrm{p}<0,001)$, siendo la RAR de $10(3,1-17,0)$, la RRR de 57,9 $(20,3-$ $77,7)$ y el NNT fue de 10 (6-33), con un intervalo de confianza del 95\%. Conclusiones: De acuerdo a la clasificación GRADE, ambos estudios nos arrojan una calidad de evidencia alta. Por lo tanto, según la bibliografía consultada, los AGHO pueden constituir una medida preventiva efectiva en las UPP y, en el caso de no evitarlas, retarda el tiem-

\section{INTRODUCCIÓN}

Las heridas crónicas, y en especial las úlceras por presión, constituyen hoy en día uno de los problemas de salud más importantes de nuestro entorno desde el punto de vista social, económico, laboral y ético-legal (1). En no pocas ocasiones, la aparición de una úlcera es vivida por el enfermo como una "humillación" ya que es una muestra clara de su deterioro físico (2). Así, el Plan de Calidad del Sistema Nacional de Salud (3) insta a minimizar las úlceras por presión, al considerarlo un proceso que perturba y pone en riesgo la seguridad de los pacientes. En la actualidad, nadie duda de que la mejor herramienta es, y debería ser, la prevención, siendo evitables hasta en un 95\% de los casos usando con coherencia los recursos correctos (4-7).

Los aceites esenciales han estado ligados históricamente al cuidado de la piel, encontrando las primeras referencias escritas hace más de 3000 años a.C. (8). Desde el año 1995 se vienen comercializando en el merca- 
po de su aparición. Aunque, dado el escaso número de estudios experimentales llevados a cabo, estimamos necesario la realización de nuevos ensayos clínicos que lo confirmen.

\section{PALABRAS CLAVE}

Úlceras por presión, ácidos grasos, eficacia, evidencia.

\section{SUMMARY}

Objectives: To know the scientific evidence about byperoxygenated fatty acid effectiveness in the prevention of the pressure ulcers and to evaluate, through the bibliography consulted, their clinical effectiveness. Material and method: There was made a compilation of information in the Cochrane library, The Joanna Briggs Institute, Pubmed-medline and Cuiden. There were only selected clinical trials, for which evaluation it has been followed the recommendations of Critical Apraisal Skill Program together with GRADE system in order to value the quality of the evidence and the strength of the recommendation. Results: No documents have been found in the Cochrane library, whereas in The Joanna Briggs Institute has been found a bestpractice where there is no reference to the hyperoxygenated fatty acid. In the rest of the databases just two randomised clinical trials that followed CASP criteria were found.In the Gallart et al study over a sample of 192 patients an UPP incidence of $19 \%$ was found in the intervention group it was found a pressure ulcer rate of $19 \%$ against the $35 \%$ in the placebo group $(p<0,007)$ being the RAR of $16(3,9-28,1)$ the $R R R$ of 45,7 (11,8 -66,6) and the NNT was of 7(4-26) with a confidence interval of 95\%.In the Torra i Bou, et al. study over a sample of 331 patients an UPP incidence of $7,32 \%$ in the intervention group against the $17,37 \%$ in the placebo group ( $p<0,001)$ being the RAR OF $10(3,1-17,0)$ the RR of 57,9 (20,3-77,7) and the NNT was of 10 (6-33) with a confidence interval of $95 \%$. Conclusions: According to GRADE classification both studies show us a high quality evidence. So according to information found, the hydrogenated fatty acids could be an effective preventive measure in pressure ulcers, and when they are not able to avoid them, they could delay their occurrence. Although there are no many clinical studies we thought necessary to carry out new clinical trials to confirm it.

\section{KEY WORDS}

Pressure ulcers, fatty acid, effectiveness, evidence.

do español productos a base de ácidos grasos hiperoxigenados (AGHO) para la prevención de úlceras por presión (UPP), con una utilización muy desigual en los diferentes niveles asistenciales (9). Los AGHO son un producto compuesto a base de ácido linoleico, ácido gamma linolénico, ácido oleico, ácido palmitito, ácido esteárico, ácido palmitoleico, ácido araquidónico y ácido eicosenoico con extractos de Equisetum arvense, familiarmente conocido como cola de caballo, e Hypericum perforatum o hierba de San Juan. Su acción está basada, según el fabricante (Laboratorio Bama-Geve) en la mejora en lo posible de la resistencia de la piel, restaurar la circulación capilar y contrarres- tar el efecto de los radicales de oxígeno producidos durante la hiperemia reactiva (10).

En este sentido, la GNEAUPP, en 2003, dentro de sus directrices generales sobre prevención de UPP, recomendaba "valorar la posibilidad de utilizar productos con AGHO en las zonas de riesgo de desarrollo de úlceras por presión" (11), recomendando la pulverización de AGHO, 2 ó 3 veces al día, sobre las zonas de riesgo, extendiendo el producto con la yema de los dedos hasta su total absorción. Al tratarse de un producto de muy rápida absorción, permite no dificultar el trabajo del profesional sanitario y de los familiares y/o cuidadores de los pacientes en su domicilio (12).
Existe suficiente evidencia de que los hallazgos de la investigación son inadecuadamente diseminados, así como que datos relevantes, productos de investigaciones no son utilizados en la práctica. En la práctica asistencial diaria, las enfermeras se encuentran cada día ante la necesidad de tomar decisiones de manera constante que afectan a la calidad de vida de los ciudadanos (13).

Por eso y ante la premisa fundamental de que la asistencia sanitaria que deberían recibir los usuarios de los sistemas sanitarios debería ser aquella que agrupa la eficacia, la efectividad y la eficiencia en su prestación. Incorporando todas aquellas prácticas que hayan demostrado ser efectivas para aquello que se pretende solucionar y excluyendo todas aquellas actuaciones que puedan no ser efectivas $\mathrm{o}$ incluso perjudiciales (14-16).

Desde la Gerencia de Atención Primaria de Albacete (SESCAM) decidimos comenzar un proceso de actualización del catálogo de productos y medidas terapéuticas suministrados a nuestros equipos de atención primara, desde una perspectiva de Enfermería Basada en la Evidencia, comenzando por los AGHO.

\section{OBJETIVOS}

- Conocer la eficacia de los ácidos grasos hiperoxigenados en la prevención de las úlceras por presión.

- Determinar el nivel de evidencia del uso de los ácidos grasos hiperoxigenados en la prevención de las úlceras por presión.

\section{MÉTODO}

Naturaleza y tipo de estudio

Revisión bibliográfica de la literatura científica sobre los AGHO en las principales bases de datos y centros de evidencia actuales.

\section{Búsqueda bibliográfica}

Tras la formulación de la pregunta: ¿son eficaces los ácidos grasos hiperoxigenados para la prevención de las úlceras por presión?, se realizó una búsqueda bibliográfica en los siguientes centros de evidencia científica: Cochrane library y The Joanna Briggs Ins- 
titute; y como bases de datos fueron utilizadas: Pubmed-medline, CINAHL y Cuiden. No hubo restricciones en fechas de publicación, es decir, desde el comienzo de la indicación hasta agosto 2007, idioma o lugar de la investigación. Como descriptores, de acuerdo a sus tesauros respectivos, fueron utilizados y dependiendo de las normas de cada base de datos, en español e inglés: "Prevención AND (\&) úlceras por presión", "Ácidos grasos hiperoxigenados", y "Prevención AND (\&) úlceras por presión AND (\&) ácidos grasos hiperoxigenados").

\section{Criterio de selección \\ de los estudios}

Como criterio de selección, nos decantamos por ensayos clínicos controlados. Para ello se procedió a la revisión de la naturaleza y tipo de estudio de la literatura científica, no siendo utilizado ningún tipo de limitador o clinical trial como descriptor.

\section{Análisis de la validez}

Los ensayos clínicos fueron evaluados siguiendo las recomendaciones del Critical Appraisal Skills Programme (CASPe) (17), que tiene como misión proporcionar habilidades a los decisores en encontrar y evaluar críticamente las mejores pruebas científicas en las que sustentar las decisiones.

Para la valoración de la calidad de la evidencia y la fuerza de las recomendaciones se optó por el sistema GRADE (Grading of Recommendations Assessment, Development and Evaluation Working Group) (18).

Con fecha 16 de agosto de 2007 , no aparece ningún documento en la Cochrane Library. En The Joanna
Briggs Institute for Evidence Based Nursing and Midwifery aparece un bestpractice relacionado con la prevención de las upp (19), traducido por el centro colaborador para España del Instituto Joanna Briggs con fecha $3 / 3 / 06$, donde no se hace ninguna referencia a los AGHO. En PUBMEDMEDLINE aparecen 3.340 documentos sobre prevención de UPP, y de éstos solamente 4 hacen referencia a prevención de UPP y AGHO, lo que supone el $0,12 \%$ del total de lo publicado en relación a la prevención. De estos 4 documentos, sólo 1 es un ensayo clínico aleatorizado, lo que supone que solamente un $0,02 \%$ del total de artículos publicados hace referencia a través de un ensayo clínico a la prevención de las úlceras por presión mediante el uso de los ácidos grasos hiperoxigenados.

En CUIDEN aparecen 107 documentos sobre prevención de UPP, 4 sobre AGHO, y los 4 hacen referencia a prevención de UPP y AGHO, lo que supone el 3,73\%. De estos 4 documentos, sólo 2 constituyen ensayos clínicos aleatorizados (1,87\%) (Tabla 1).

En función de todos los criterios anteriormente reseñados fueron seleccionados los siguientes artículos:

Gallart E, Fuentesaz C, Vivas G, Garnacho I, Font L, Arán R. Estudio experimental para comprobar la efectividad de los ácidos grasos hiperoxigenados en la prevención de las úlceras por presión en pacientes ingresados. Rev Enferm Clínica. 2001; 11(5): 179-183.

Torra i bou JE, Segovia T, Verdú J, Nolasco A, Rueda J, Arboix i Perejamo M. The effectiveness of hyperoxygenated fatty acid compound in preventing pressure ulcers. J Wound Care. 2005; 14(3): 117-121.

El artículo de Gallart, et al. obtuvo una puntuación de 4 puntos en el CASPe, lo que valida su utilización como fuente. La incidencia de úlceras por presión en el grupo control fue del $35 \%$ (IC 95\%, 21-34\%) y en el grupo experimental del 19\% (IC 12-29\%), siendo esta diferencia estadísticamente significativa $(p=0,007)$. Los autores estudiaron también el tiempo que tardaban en aparecer las lesiones mediante el cálculo de la mediana. Así, en el grupo control la mediana fue de 3 días (mín. 3; máx. 5), mientras que en el grupo experimental fue de 5 días (mín. 3; máx. 7), siendo esta diferencia estadísticamente significativa $(\mathrm{U}=218,5$ $\mathrm{p}=0,025)$. Con un intervalo de confianza del $95 \%$ el tratamiento experimental reduce la tasa de un evento no deseado (reducción absoluta del riesgo) en 16,0 $(3,9-28,1)$, la reducción relativa del riesgo (RRR) es de 45,7 (11,8$66,6)$ y el número de pacientes que es necesario tratar con el tratamiento experimental para conseguir un evento adicional con respecto a los que se conseguirían con el tratamiento control (NNT) se situó en 7 (4-26).

Por su parte, en el estudio de Torra i bou, et al., obtuvo una puntuación 5 en el CASPe. La incidencia de úlceras por presión en el grupo intervención fue del $7,32 \%$, y en el grupo control del $17,37 \%$, siendo esta diferencia estadísticamente significativa $(\mathrm{p}=0,006)$; las curvas de supervivencia (Kaplan-Meier) y los modelos de regresión muestran una diferencia estadísticamente significativa para ambos grupos $p \leq 0,001$. Con un intervalo de confianza del $95 \%$, el trata-

\begin{tabular}{|c|c|c|c|c|c|}
\hline \multirow[b]{2}{*}{ Descriptores } & \multicolumn{5}{|c|}{ Bases de datos y fuentes } \\
\hline & $\begin{array}{l}\text { Cochrane } \\
\text { Library }\end{array}$ & $\begin{array}{l}\text { Joanna Briggs } \\
\text { Institute }\end{array}$ & PUBMED & CUIDEN & CNHAL \\
\hline Úlceras por presión & 314 & 2 & 7.750 & 352 & 0 \\
\hline Prevención \& úlceras por presión & 30 & 1 & 3.440 & 107 & 982 \\
\hline Ácidos grasos hiperoxigenados & 30 & 0 & 4 & 4 & 4 \\
\hline Prevención \& úlceras por presión & & & & & \\
\hline$\&$ ácidos grasos & 0 & 0 & 3 & 3 & 4 \\
\hline
\end{tabular}




\begin{tabular}{|c|c|c|c|}
\hline \multicolumn{4}{|c|}{ Tabla 2. Comparación de estudios seleccionados } \\
\hline Estudios & RAR (IC 95\%) & RRR (IC 95\%) & NNT (IC 95\%) \\
\hline $\begin{array}{l}\text { Torra i bou, et al. } \\
\text { Gallart, et al. }\end{array}$ & $\begin{array}{l}10,0(3,1-17,0) \\
16,0(3,9-28,1)\end{array}$ & $\begin{array}{l}57,9(20,3-77,7) \\
45,7(11,8-66,6)\end{array}$ & $\begin{array}{l}10(6-33) \\
7(4-26)\end{array}$ \\
\hline
\end{tabular}

miento experimental reduce la tasa de un evento no deseado (reducción absoluta de riesgo, RAR) en 10,0 ( 3,1 $17,0)$, la reducción relativa del riesgo (RRR) es de 57,9 (20,3-77,7) y el número de pacientes que es necesario tratar con en tratamiento experimental para conseguir un evento adicional con respecto a los que se conseguirían con el tratamiento control (NNT) se situó en 10 (6-33). En la Tabla 2 podemos encontrar la comparación entre los resultados obtenidos en ambos estudios.

En relación a nuestra intención de determinar el nivel de evidencia del uso de los ácidos grasos hiperoxigenados como medida preventiva de las úlceras por presión, de acuerdo a las recomendaciones establecidas por el sistema GRADE. El estudio de Gallart et al. es un Ensayo clínico aleatorizado (evidencia alta), con una limitación importante por no cegamiento $(-1)$. Consistente (=), evidencia directa $\mathrm{RR}>5(+2)$. Con una calificación final de evidencia alta. Por su parte, el estudio de Torra i bou, et al. es un ECA (evidencia alta) sin limitaciones importantes, consistente $(=)$ y con una evidencia reforzada por la presencia de $R R>5$. Por lo tanto, nos arroja una calificación final de evidencia alta.

\section{DISCUSIÓN}

Para la realización del estudio nos decantamos exclusivamente por ensayos clínicos controlados, ya que, como experimentos controlados que son, apor- tan mayores niveles de evidencia científica, hasta el punto de que muchos autores consideran que constituye "el ideal de la ciencia" (20-22). Mediante las contribuciones científicas de tales diseños, la práctica de la enfermería puede estar asentada firmemente en la investigación, como sostiene un gran número de estudiosos y académicos en la actualidad (23). Sin embargo, la aplicación de este criterio de selección motivó que el número de estudios fuese tan reducido, desistiendo en la idea original de realizar un metaanálisis.

Consideramos que la calidad metodológica de ambos estudios analizados es muy alta. El estudio de Gallar, et al. consiste en un ensayo clínico controlado y aleatorizado sin cegamiento, realizado en las unidades de hospitalización del Hospital General Vall d'Hebron, y en el caso de Torra i Bou se trata de un estudio multicéntrico doble ciego, llevado a cabo en... Ambos estudios están claramente orientados a una pregunta bien definida; en el de Gallart, et al. se ha pretendido identificar si existen diferencias significativas en la incidencia de úlceras por presión entre los enfermos a los que se realiza la prevención con ácidos grasos hiperoxigenados y los que no los reciben. Por su parte, Torra i Bou y su equipo han comparado los efectos de un compuesto de ácidos grasos hiperoxigenados $\left(\mathrm{Mepentol}^{\circledR)}\right.$ frente a un placebo oleoso en la prevención de las úlceras por presión. Este hecho, desde nuestro punto de vista, contribuye a la diferenciación, como producto terapéutico, de los ácidos grasos hiperoxigenados frente a las diferentes soluciones oleosas existentes. Ambos estudios consideraron adecuadamente a todos los pacientes que fueron incluidos en el mismo, estando bien descritas las pérdidas. Los grupos fueron similares al comienzo de ambos ensayos, si bien es cierto que los grupos de Torra i Bou era superiores numéricamente a los de Gallart (190 frente a 96).

$\mathrm{Al}$ margen de la intervención en estudio, los grupos fueron tratados de igual modo y los resultados fueron medidos en función de la aparición o no de úlceras por presión, tras comprobar la homogeneidad de ambos grupos. Con todo ello, podríamos afirmar que es elevada la validez interna en los estudios analizados. Con respecto a su validez externa o aplicabilidad de los resultados en la práctica clínica, consideramos que, al ser los pacientes estudiados muy similares a los atendidos en nuestro medio, sus resultados son generalizables y podría recomendarse el uso de los ácidos grasos hiperoxigenados como medida preventiva.

\section{CONCLUSIONES}

Según la evidencia disponible, procedente de dos ensayos clínicos, los ácidos grasos hiperoxigenados podrían constituir una medida preventiva eficaz en las UPP, y, en caso de no ser evitarlas, retardar el momento de aparición.

De acuerdo a la clasificación GRA$\mathrm{DE}$, ambos estudios analizados arrojan una calidad de evidencia alta. De hecho, esta recomendación preventiva en el caso de las UPP aparece en las diferentes guías de práctica clínica y protocolos consultados (23).

\section{BIBLIOGRAFÍA}

1. Soldevilla JJ, Navarro S, Roselle C, Sarabia R, Vals G. Problemática de las úlceras por presión y sus repercusiones legales. Madrid: SPA; 2004.

2. Gerencia de Atención Primaria Albacete (SESCAM). Heridas crónicas y úlceras por presión: guía de prevención y tratamiento. Albacete 2007.

3. Ministerio de Sanidad y Consumo. Plan de calidad para el Sistema Nacional de Salud. Madrid 2006.

4. Herrero E, Torra i Bou JE, Martínez M. Utilización de un colchón alter- nante de aire en la prevención y el tratamiento de úlceras por presión en un paciente de atención domiciliaria. Gerokomos. 2000; 11 (2): 95-101.

5. Verdú J, López P, Fuentes G, Torra i Bou JE. Prevención de UPP en talones. Impacto clínico y económico en una unidad de medicina interna. Rev ROL Enfermería. 2004; 27: 60-4.

6. Soldevilla JJ, Torra i Bou JE, Verdú J, Martínez F, López P, Rueda López J, Mayán JM. 2 Estudio Nacional de Prevalencia de Úlceras por Presión en España, 2005. Epidemiología y variables definitorias de las lesiones y pacientes. Gerokomos. 2006; 17 (3): 154-72. 
7. Camiah S. Utilization of nursing reseach in practice and application strategies to raise research awarenes amongst nurse practitioners: a model for sucess. Journal of advanced nursing. 1997; 26: 1193-202.

201 8. Baker J. Essential oils: a complimentary therapy in wound managament. J Wound Care. 1998; 7: 355-60.

9. García FP, Pacorbo PL, López J. Recursos materiales para la prevención y el tratamiento de la úlceras por presión: análisis de la situación en Andalucía. Gerokomos. 2006; 17 (1): 47-57.

10. Torra i Bou JE, Rueda J, Segovia T, Bermejo M. Aplicación tópica de un compuesto de ácidos grasos hiperoxigenados. Rev ROL Enf. 2003; 26 (1): 54-61.

11. Grupo Nacional para el estudio y asesoramiento en úlceras por presión y heridas crónicas (GNEAUPP). Directrices generales sobre prevención de las úlceras por presión. Logroño 2003.

12. Dossier científico Mepentol. Barcelona: Laboratorios Bama-Geve SA, 2002.

13. Barderas A, Escobar G. Fuentes de información en Enfermería: buscando la evidencia. Rev Enferm en Anestesia-Reanimación y terapia del dolor. 2004; 12: 43-7.

14. Martínez JR. Barreras e instrumentos facilitadores de la enfermería basada en la evidencia. Rev Enfermeria Clínica. 2003; 13: 303-8.

15. Barderas A, Escobar G, Jones C. Documentación y producción científica española en: Medicina y Enfermería basada en la evidencia. 2004. En prensa.

16. Fuentesalz C, Moreno MT, Martín MC, Comet P, Uriel P, Orts I, Jiménez
MI. Glosario de términos para la práctica clínica basada en la evidencia. Rev Enferm Clin. 2002; 12 (4): 173-81.

17. Castañar M, Martínez V, Espí JJ. Critical Appraisal Skills Programme (CAS$\mathrm{Pe}$ [http://www.redcaspe.org]. [Consultado el 12 septiembre 2007]. Disponible en: http://www.redcaspe.org/porque/lecturacritica.htm.

18. Marzo M, Alonso P, Roaeche R. ¿¿Cómo clasificar la calidad de evidencia y la fuerza de las recomendaciones? Rev. Aten Primaria 2006; 37 (1): 5-8.

19. Lesiones por presión. Primera parte: prevención de las lesiones por presión.Best Practice. 1997; 1 (1): 1-6. [Consultado el 16 agosto2007]. Disponible en www.isciii.es/htdocs/redes/investen/pdf/jb/1997_1_1_Úlceras.pdf.

20. Polit DF, Hungler BP. Investigación científica en ciencias de la salud. $6^{\mathrm{a}}$ ed Mexico: McGraw-Hill; 2000.

21. López IM. Prevención de complicaciones en enfermos coronarios por profesionales de enfermera de atención primaria. Index de Enfermeria. 2002; XI (36-37): 60-63. [Consultado el 16 de agosto de 2007]. Disponible en: http://www.index-f.com/index-enfermeria/revista.php.

22. Cabrero J. Enfermería basada en la evidencia y utilización de la investigación. Index de Enfermería. 1999; 27: 12-18. http://www.index-f.com/ciberindex.php?l=3\&url=/index-enfermeria/27revista/27_articulo_12-18.php.

23. García J, Mateo A, Herrero JV, Pómer M, Masoliver A, Lizán L. Guía Fisterra UPP. Prevención, tratamiento y derivación en úlceras por presión y vasculares en atención primaria. de salud. [Consultado el 16 agosto 2007]. Disponible en: http://www.fisterra.com/guias2/upresion.asp. 